\title{
Distribución corporal de garrapatas (Acari: Ixodidae y Argasidae) asociadas a Odocoielus virginianus (Artiodactyla: Cervidae) y Ovis canadensis (Artiodactyla: Bovidae) en tres estados del norte de México
}

Mariana Cuesy León ${ }^{\text {a }}$

Zinnia Judith Molina Garza ${ }^{\mathrm{a}^{*}}$

Roberto Mercado Hernández ${ }^{\text {a }}$

Lucio Galaviz Silva ${ }^{\text {a }}$

${ }^{a}$ Universidad Autónoma de Nuevo León, Facultad de Ciencias Biológicas, Ave. Universidad S/N, Ciudad Universitaria. 66455 San Nicolás de los Garza, Nuevo León. México.

*Autor de correspondencia: zinnia.molinagr@uanl.edu.mx; molinazinnia@hotmail.com

\section{Resumen:}

Las garrapatas impactan como vectores por transmitir patógenos de importancia médica y veterinaria en México, pero los estudios taxonómicos de abundancia, prevalencia, intensidad y preferencia en la distribución corporal de venado cola blanca (Odocoileus virginianus) y borrego cimarrón (Ovis canadensis) son precarios, por lo cual estos fueron los objetivos del presente trabajo en Sonora, Nuevo León y Tamaulipas, México. El área de estudio abarcó ranchos cinegéticos autorizados donde se practica la cacería. Se examinaron $233 O$. virginianus y cuatro $O$. canadensis, recolectándose 372 garrapatas [21 ninfas $(5.65 \%)$ y 351 adultos (94.35\%)]; $41 \%$ fueron hembras y $59 \%$ machos. Las garrapatas presentes en $O$. virginianus fueron Otobius megnini, Rhipicephalus (Boophilus) microplus y Dermacentor (Anocentor) nitens, mientras que Dermacentor hunteri fue la única en O. canadensis. Las orejas fue la región más infestada (83 hembras, 70 machos y 21 ninfas, $46.77 \%$ en total) y la menos infestada fueron las piernas (10 machos y nueve hembras, $5.1 \%$ ), con diferencia significativa $(P<0.005)$. Este estudio reporta por primera vez la abundancia, intensidad y 
prevalencia de garrapatas en $O$. virginianus en el norte de México y particularmente en los estados de Tamaulipas y Nuevo León, pues solo las garrapatas de O. canadensis habían sido reportadas en Sonora. Estos resultados indican que, aunque los ungulados están en semicautiverio, es importante controlar la infestación por garrapatas de acuerdo a los sitios de adherencia preferidos para aplicar los tratamientos acaridas, debido a la importancia como vectores en la transmisión de patógenos.

Palabras clave: Venado cola blanca, Borrego cimarrón, Garrapatas, Rhipicephalus microplus, Otobius megnini, Dermacentor nitens, Dermacentor hunteri.

Recibido: 26/02/2019

Aceptado:20/11/2019

\section{Introducción}

Las garrapatas son ectoparásitos de anfibios, reptiles, aves y mamíferos, hematófagos en todos sus estadios ${ }^{(1)}$. Debido a sus hábitos alimenticios, causan efectos directos en sus hospederos como escasa ganancia de peso, acción traumática, tóxica, infecciosa o expoliadora y también, efectos indirectos que ocasionan deterioro de piel y muerte por enfermedades dérmicas ${ }^{(2,3)}$. Durante su ciclo de vida, la garrapata puede adquirir patógenos de forma horizontal o vertical ${ }^{(4)}$ y transmitir una amplia gama de microorganismos de importancia médica y veterinaria como Babesia spp., Borrelia spp., Anaplasma phagocytophilum y Rickettsia spp., considerándose como vectores de importancia mundial, superados únicamente por los mosquitos ${ }^{(5)}$.

La fauna silvestre constituye un componente en el ciclo de transmisión del triángulo vectorhospedero-patógeno, donde frecuentemente se incluye al humano como hospedero accidental, convirtiéndose en un ciclo zoonótico ${ }^{(6)}$. Por lo tanto, la prevalencia de enfermedades nuevas y re-emergentes transmitidas por garrapatas, conforman un problema de salud pública en el mundo ${ }^{(7)}$. La distribución geográfica de las garrapatas está influenciada principalmente por las variaciones climáticas y geográficas, el tipo de vegetación, el paisaje agrícola, la dinámica poblacional de sus hospederos silvestres ${ }^{(3)}$; así como el movimiento ilegal de ganado y de Odocoileus virginianus para comercialización sin cumplir normas sanitarias ${ }^{(8)}$, los cuales son factores que facilitan su dispersión hacia sitios donde no se encontraba naturalmente, aumentando en el humano el riesgo de exposición para adquirir enfermedades asociadas a estos vectores ${ }^{(9,10)}$. Los estudios de garrapatas reportan preferencias específicas de Rhipicephalus annulatus por cérvidos ${ }^{(11)}$, así como especificidad de Dermacentor spp. e Ixodes spp. por cabeza y cuello ${ }^{(12)}$. 
En México, se han identificado 77 especies de garrapatas, de las cuales en la ganadería nacional las de importancia por los daños directos e indirectos son $R$. (Boophilus) microplus, B. anulatus, Amblyomma cajennense, A. imitador. A. maculatum, A. triste, A. americanum y Anocentor nitens, sin embargo, las que sobresalen por su mayor impacto económico son $R$. microplus y A. cajennense, ${ }^{(13,14)}$ con pérdidas por US \$ 573'608,076 ${ }^{(15)}$. En los estados de Sonora, Nuevo León y Tamaulipas, se ha aprovechado la presencia de $O$. virginianus y Ovis canadensis para aumentar los ingresos a través de la caza legalizada que se realiza en ranchos cinegéticos. En 1996, se inició en el Rancho El Plomito, Sonora, el programa de conservación que consistió en la construcción de un encierro para la reproducción de especies de importancia económica en condiciones de semi-cautiverio en una superficie de 961 ha. Para el 2014, se contó con una población reproductiva de $O$. canadensis, $O$. v. couesi y $O$. hemionus para realizar las primeras repoblaciones por la Organización de Vida Silvestre (OVIS, AC) ${ }^{(16)}$. Las autoridades mexicanas reformaron la operatividad de los ranchos cinegéticos mediante la implementación del actual sistema de Unidades de Manejo para la Conservación de la Vida Silvestre (UMA), que permite la conservación y ordenación de la fauna silvestre en su hábitat natural, así como el aprovechamiento racional de poblaciones y ejemplares de especies silvestres o en semi-cautiverio ${ }^{(17,18)}$. En las localidades estudiadas está permitido la caza regulada de estos ungulados, pero los estudios poblacionales de ectoparásitos, su distribución corporal y la presencia de especies de interés cinegético son escasos $^{(3,12)}$. Por lo cual, los resultados obtenidos con el presente estudio permitirán planificar las zonas del cuerpo donde se aplicarán los tratamientos o dispositivos acaricidas para su control, que han demostrado éxito en su erradicación ${ }^{(12)}$. Los objetivos de este estudio fueron i) identificar taxonómicamente las especies de las garrapatas, ii) determinar su prevalencia, iii) estimar la abundancia e intensidad y iv) describir la distribución corporal en $O$. virginianus y $O$. canadensis en ranchos cinegéticos de Sonora, Nuevo León y Tamaulipas en la región norte de México. La información obtenida ayuda a comprender el riesgo potencial de las garrapatas como vectores de enfermedades de importancia y establecer medidas preventivas y correctivas.

\section{Material y métodos}

\section{Localidades estudiadas}

El presente estudio se realizó del año 2014 al 2018 en distintas localidades del estado de Tamaulipas, Nuevo León y Sonora, ubicados en el norte de México, en los meses de octubre a febrero, período de caza legal en UMAs in situ o ex situ de especies como $O$. virginianus y O. canadensis $^{(17,18)}$. Dos localidades se ubican en la Sierra Madre Occidental en el estado de Sonora: Rancho El Aigame (registro de la UMA: DGVS-CR-EX-1271-SON) municipio La Colorada (28 43' 41" N, 110 2'0.65" W) a 400 msnm y Rancho El Pitiquito (registro de la

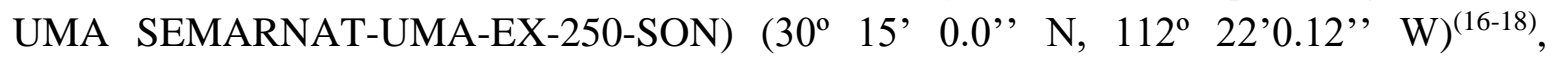


municipio El Pitiquito, donde predomina el clima seco y semiseco con precipitación media de $450 \mathrm{~mm}$ anuales ${ }^{(19)}$. En el estado de Nuevo León, las colectas se realizaron en el Rancho Mamulique (clave de registro DFYFS-CR-EX-0333-NL), municipio de Salinas Victoria (26 ${ }^{\circ}$ 7' $0.59^{\prime}$ ' $\mathrm{N}, 100^{\circ} 19^{\prime} 0.58^{\prime}$ 'W), a $464 \mathrm{msnm}$, con clima semicálido y seco estepario caliente; cuenta con una temperatura media anual de $21-23{ }^{\circ} \mathrm{C}$ y precipitación media anual de 380 $\mathrm{mm}^{(19)}$. En el estado de Tamaulipas, las colectas se realizaron en dos localidades, una en el Rancho Santa Clara (registro de la UMA DGVS-CR-EX-1819-TAM), Nuevo Laredo (27 33'0.11' ' N, 99 47' 59.9', W), con clima caracterizado por ser el más seco y extremoso del estado, con oscilaciones que varían desde $-14{ }^{\circ} \mathrm{C}$ en invierno y hasta los $40{ }^{\circ} \mathrm{C}$ en verano; su precipitación media anual es de $472.5 \mathrm{~mm}^{(19)}$. La segunda localidad fue Rancho Los Columpios (clave de registro DGVS-CR-EX-2066-TAM), municipio de Guerrero (26 $33^{\prime}$ $18^{\prime}$ ' N, 99 $22^{\prime}$ ' $0.37^{\prime}$ ' W) ubicado sobre la cuenca del río Bravo, Tamaulipas. Su clima se considera de tipo seco, muy cálido y con una precipitación media anual de 440 mm ${ }^{(18-20)}$.

\section{Colecta e identificación taxonómica de garrapatas}

Todos los ejemplares de garrapatas se obtuvieron de ejemplares cazados de $O$. virginianus y $O$. canadensis durante la temporada de caza, a través de la autorización emitida por la Secretaría del Medio Ambiente y Recursos Naturales a cada UMA ${ }^{(16-18)}$. La tasa de aprovechamiento de estas especies se orienta a la caza de ejemplares machos y adultos; los cazadores, después de adquirir un paquete de cacería, fueron acompañados por técnicos del grupo OVIS ${ }^{(16)}$. Durante las colectas y utilizando pinzas estériles, las garrapatas fueron individualmente removidas de la parte superior de la cabeza, el oído, la escapula, la parte media dorsal del cuello y extremidades inferiores de los animales cazados ${ }^{(21)}$. Las garrapatas vivas se transportaron en viales de $12 \mathrm{ml}$ previamente etiquetado con fecha, hospedero, estadio, localidad y distribución corporal de la garrapata en el hospedero que contenían algodón humedecido con agua bidestilada estéril y posteriormente fueron trasladados al Laboratorio de Patología Molecular y Experimental (LPME, FCB, UANL), conservándose a $4{ }^{\circ} \mathrm{C}$. Para aquellos ectoparásitos que morían en el trayecto, se prepararon viales con alcohol etílico absoluto como preservador, evitándose el deterioro de los caracteres morfológicos para posterior identificación taxonómica ${ }^{(22)}$.

La identificación taxonómica de garrapatas se realizó con ayuda de un estereoscopio a 10X - 40X (EZ4E, Leica Microsystem, Guadalajara, Jalisco, México) y con base en claves taxonómicas específicas se determinó el género y la especie a la que pertenecen y se registró el sexo y estadio ${ }^{(23-25)}$. En la identificación taxonómica de las garrapatas se consideraron las siguientes estructuras distintivas de cada una de las especies:

Otobius megnini: Sin escudo dorsal y con gnatostoma ventral, base del capítulo recto y rectangular, ojos ausentes, hipostoma vestigial o atrofiado, tegumento con espinas, ambulacro ausente al final de las patas (Figura 1). 
Figura 1: Características taxonómicas de Otobius megnini

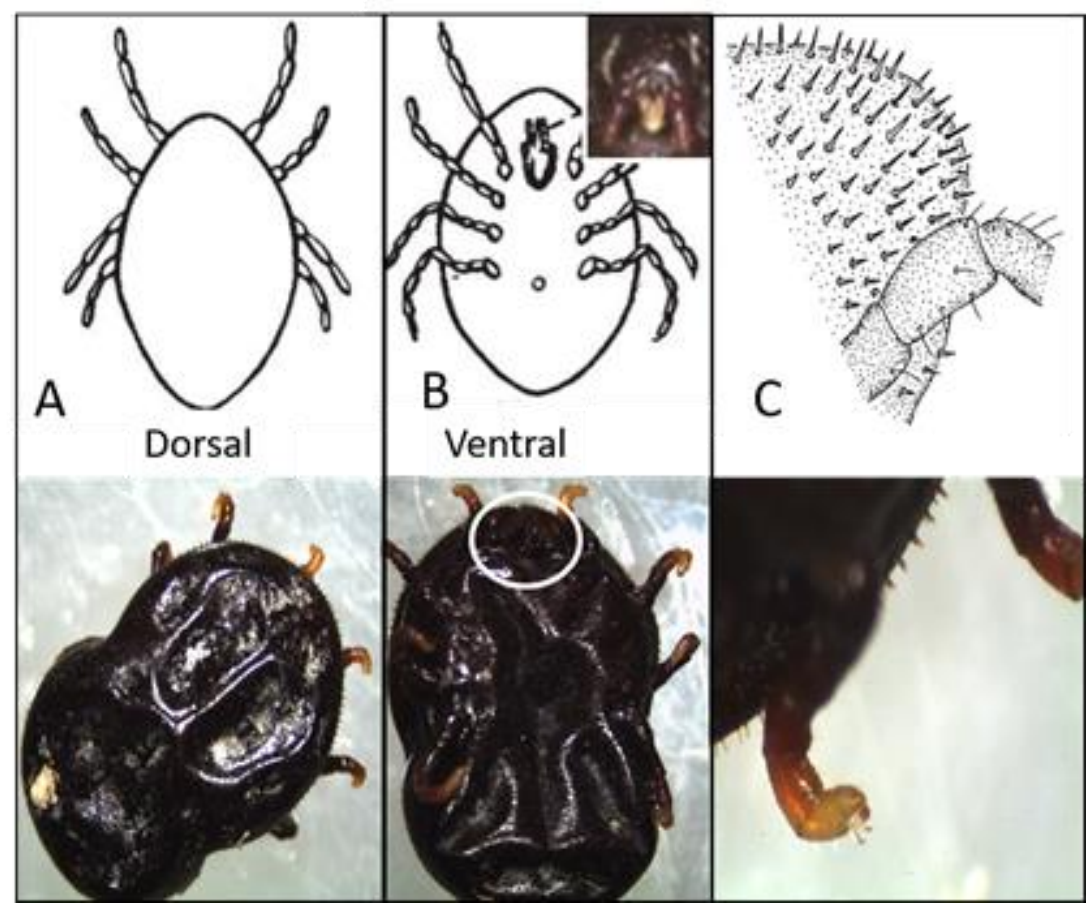

A) Vista dorsal. Diagrama y fotografía de ninfa. B) Vista ventral con detalles del gnastoma anterior, base del capítulo rectangular en el recuadro. C) Integumento con espinas (entre las espinas el integumento es liso).

Rhipicephalus (Boophilus) microplus: Los machos presentan placas ventrales y un apéndice caudal, escudo cubre la región dorsal del macho y escudo cubre la región dorsal anterior de la hembra, ojos presentes, base del capítulo hexagonal, coxa I con espina doble y la coxa IV del tamaño normal, prominente y visible desde la vista dorsal, festones ausentes (Figura 2). Las especies de Dermacentor presentan un gnastoma anterior con la base del capítulo recto y rectangular, festones, la coxa IV muy grande en machos mientras que la coxa I tiene espuelas grandes y pareadas (Figura 3). Para la diferenciación entre las especies de $D$ hunteri y $D$. nitens, se contaron el número de festones: $D$. hunteri tiene 11 y presenta un ornamento con un patrón característico, con placas espiraculares grandes y posteriores a la IV pata, en forma de anillo (Figura 4), mientras que D. nitens cuenta con siete festones (Figura 5). Todos los esquemas de las figuras fueron tomadas de las claves taxonómicas ${ }^{(24,25)}$. 
Figura 2: Características morfológicas para la identificación taxonómica de Rhipicephalus (Boophilus) microplus

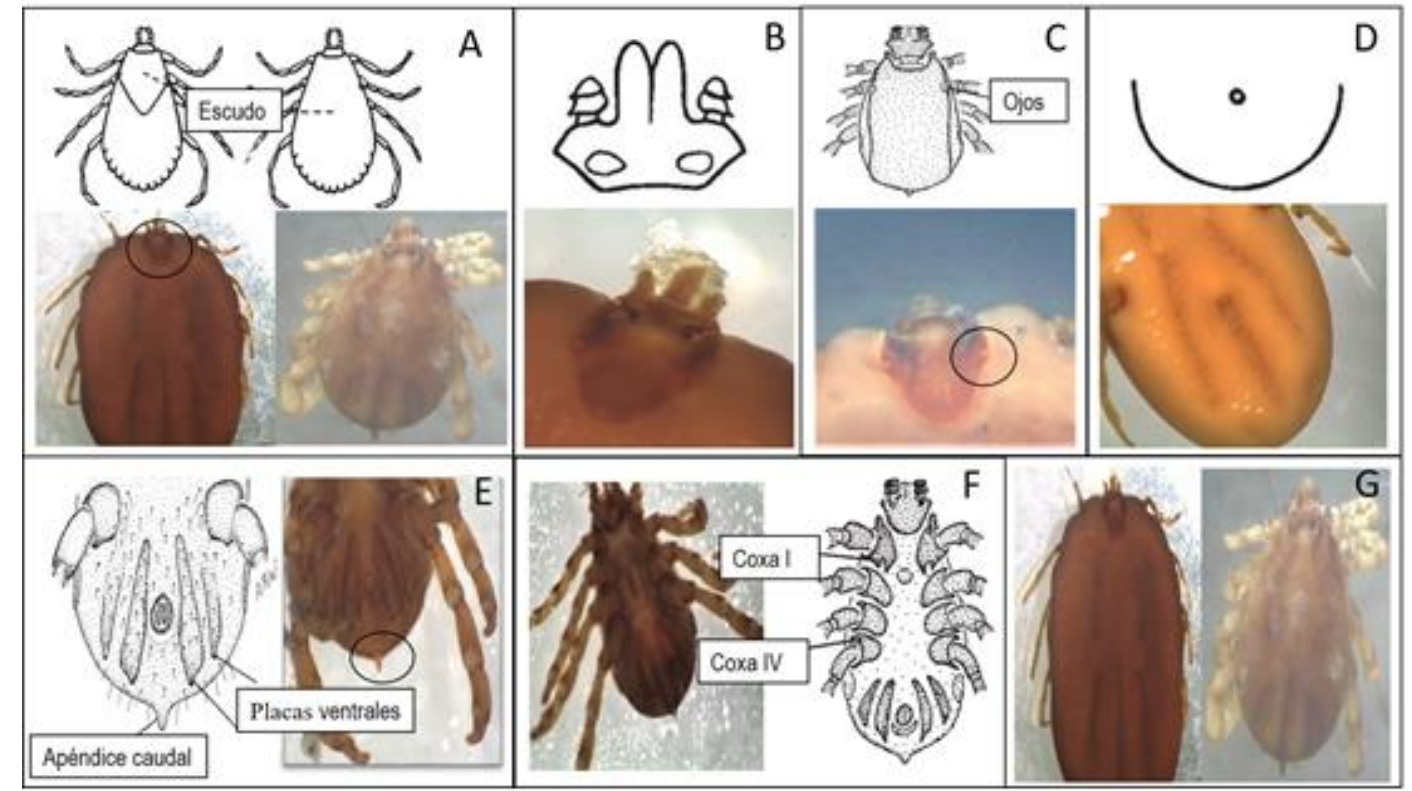

A) Escudo dorsal presente. B) Gnastoma anterior, base del capítulo con márgenes angulares. C) ojos presentes. D) Festones ausentes. E) Placas ventrales y apéndice caudal. F) Coxa I y IV. G) Ejemplares adultos hembra y macho.

Figura 3: Características morfológicas para la identificación taxonómica de Dermacentor spp

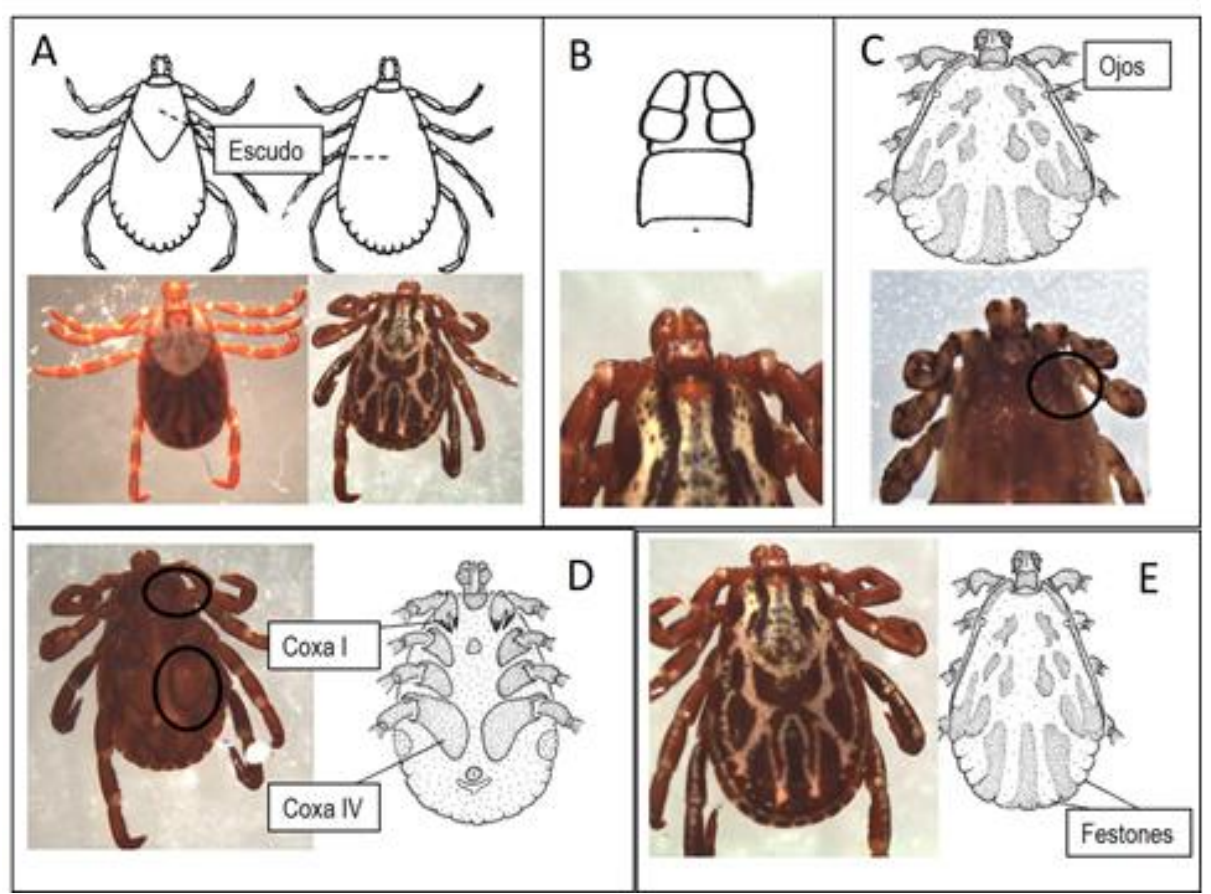

A) Escudo dorsal presente. B) Gnastoma anterior, base del capítulo recto y rectangular. C) Ojos presentes. D) Morfología de la coxa I y IV. E) Festones en extremo posterior. 
Figura 4: Características morfológicas para la identificación taxonómica de Dermacentor hunnteri

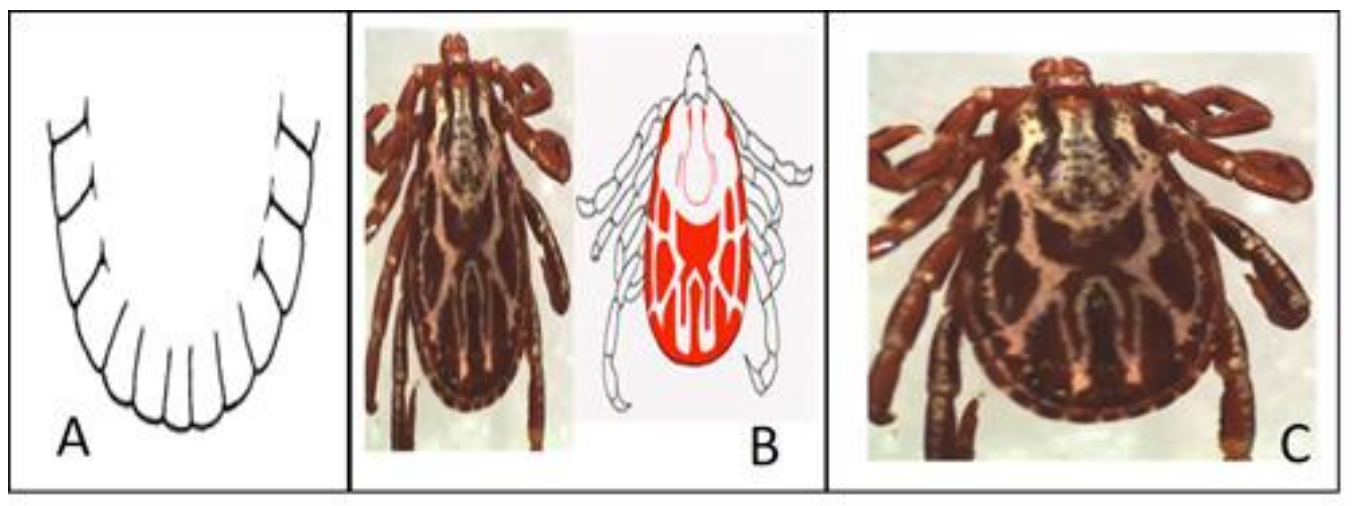

A) Once festones. B) Ornamento presente (mostrado en el patrón). C) Ejemplar (macho) completo.

Figura 5: Características morfológicas para la identificación taxonómica de Dermacentor

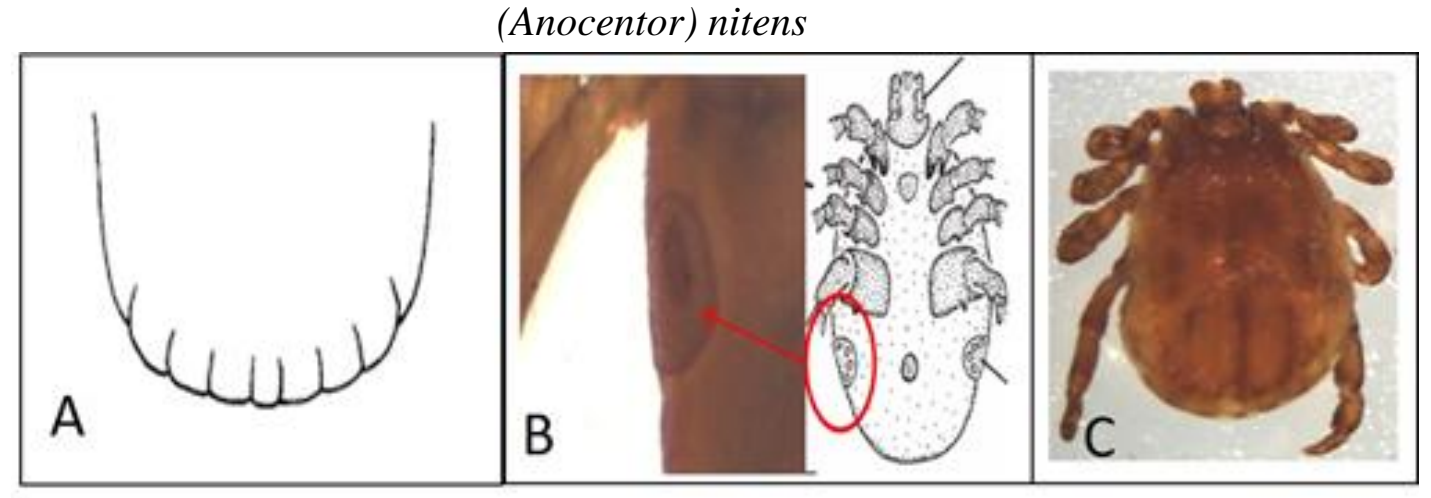

A) Siete festones. B) Placas espiraculares grandes y posteriores a la IV pata (forma un anillo). C) Ornamento ausente, ejemplar (macho) completo.

\section{Análisis estadístico}

Se calcularon la prevalencia (porcentaje de hospederos infestados por especie de garrapata), intensidad (total de garrapatas/hospederos infestados por cada especie de garrapata), abundancia (total de garrapatas por especie/total de hospederos) y la proporción de sexos por especie de garrapata ${ }^{(26)}$. Se determinó la asociación significativa entre el vector, la ubicación del hospedero, sexo y localidad de colecta de las garrapatas a partir de una prueba de Chi cuadrada $\left(\mathrm{X}^{2}\right)$ a un nivel de significancia del $95 \%$ del intervalo de confianza inferior y superior (IC). Adicionalmente, los datos se analizaron con la prueba $Z$ para comparar proporciones poblacionales entre estadios, especies de garrapatas, localidades y hospederos con el programa del SPSS versión $17^{(12)}$. 


\section{Resultados}

Se inspeccionaron un total de 237 hospederos, de los cuales 233 fueron ejemplares de $O$. virginianus y cuatro de $O$. canadensis, los porcentajes de infestación corporal fueron $17.16 \%$ y $100 \%$, respectivamente. Se colectó un total de 372 garrapatas, $5.65 \%$ ninfas y 94.35\% adultas. Las garrapatas se identificaron en cuatro especies distintas, tres pertenecientes a la familia Ixodidae: Rhipicephalus (Boophilus) microplus (Canestrini, 1887), Dermacentor (Anocentor) nitens (Neumann, 1897) y Dermacentor hunteri (Bishopp, 1912); y Otobius megnini (Dugès 1883) de la familia Argasidae (Cuadro 1).

Cuadro 1: Identificación de garrapatas por hospedero (Odocoileus virginianus y Ovis canadensis), sexo y localidad

\begin{tabular}{|c|c|c|c|c|c|}
\hline \multirow[b]{2}{*}{ Localidad } & \multirow{2}{*}{$\begin{array}{l}\text { Hospedero } \\
(\mathbf{n} /+)\end{array}$} & \multirow[b]{2}{*}{ Especies } & \multirow[b]{2}{*}{ GE/TE (\%) } & \multicolumn{2}{|l|}{ Sexo } \\
\hline & & & & $\begin{array}{l}\mathbf{H} \\
\mathrm{n} /(\%)\end{array}$ & $\begin{array}{l}M \\
\mathbf{n} /(\%)\end{array}$ \\
\hline \multirow[t]{3}{*}{ Sonora } & $\begin{array}{l}\text { O. virginianus } \\
(16 / 6)\end{array}$ & * Otobius megnini & $19 / 204(9.3)$ & NA & NA \\
\hline & O canadonsic & *O. megnini & $2 / 204(0.98)$ & NA & NA \\
\hline & $(4 / 4)$ & $\begin{array}{l}\text { Dermacentor } \\
\text { hunteri }\end{array}$ & $183 / 204(89.7)$ & $28 /(13.7)$ & $155 /(76)$ \\
\hline \multirow[t]{2}{*}{ Nuevo León } & O. virginianus & $\begin{array}{l}\text { Rhipicephalus } \\
\text { microplus }\end{array}$ & $98 / 151(64.9)$ & $84 /(55.6)$ & $14 /(9.3)$ \\
\hline & $(202 / 28)$ & D. nitens & $53 / 151(35.1)$ & $19 /(12.6)$ & $34 /(22.5)$ \\
\hline Tamaulipas & $\begin{array}{l}\text { O. virginianus } \\
(15 / 6)\end{array}$ & R. microplus & 17/17 (100) & $13 /(76.5)$ & $4 /(23.5)$ \\
\hline \multirow{2}{*}{$\begin{array}{l}\text { Total } \\
\text { O. virginianus }\end{array}$} & $(233 / 40)$ & NA & $* 19 / 372(5.1)$ & NA & $\mathrm{NA}$ \\
\hline & & & $168 / 372(45.2)$ & $116 /(41)$ & $52 /(59)$ \\
\hline \multirow{2}{*}{$\begin{array}{l}\text { Total } \\
\text { O. canadensis }\end{array}$} & $(4 / 4)$ & NA & $* 2 / 372(0.53)$ & NA & NA \\
\hline & & & $183 / 372(49.19)$ & $28 /$ & $155 /$ \\
\hline
\end{tabular}

GE/TE= Garrapatas por especie /total del Estado (\%); n= número de ejemplares; *= ninfas; NA= no aplica.

En cuanto al sexo de las garrapatas, el $41 \%$ fueron hembras y $59 \%$ machos (Cuadro 1), pero distribuidas por sexo y por hospedero, en $O$. virginianus la mayoría fueron hembras (116/168, $69 \%$ ) con $31 \%$ machos (52/168). Por el contrario, en $O$. canadensis la mayor prevalencia de $D$. hunteri fue de machos $(155 / 183,84.7 \%)$ con respecto al $15.3 \%(28 / 183)$ de hembras, con asociación significativa entre la proporción de machos y hembras de garrapatas entre $O$. 
virginianus y $O$. canadensis $\left(\mathrm{X}^{2}=104.57\right.$, g.l. $\left.=1, P<0.05\right)$. La proporción de hembras y machos entre las cuatro especies de garrapatas en $O$. virginianus también mostró asociación significativa $\left(\mathrm{X}^{2}=39.92\right.$, g.l. $\left.=1, P<0.05\right)$, igual que entre machos y hembras por localidad $\left(\mathrm{X}^{2}=105.01\right.$, g.l.= 2, $\left.P<0.05\right)$. Esto fue consistente con el análisis de proporciones poblacionales entre estadios, especies de garrapatas, localidades y hospederos, ya que fue confirmatoria con la prueba $Z$ que mostró una asociación significativa ( $Z>1.2$, IC 95\%).

Cuadro 2: Porcentaje de la distribución corporal de las especies de garrapatas identificadas

\begin{tabular}{|c|c|c|c|c|c|c|}
\hline $\begin{array}{l}\text { Región } \\
\text { corpora }\end{array}$ & Sexo & $\begin{array}{c}\text { R. microplus } \\
\mathbf{n} /(\%)\end{array}$ & $\begin{array}{c}\text { D. nitens } \\
\mathbf{n} /(\%)\end{array}$ & $\begin{array}{c}\text { O. megnin } \\
\mathrm{n} /(\%)\end{array}$ & $\begin{array}{c}\text { D. hunteri } \\
\text { n/(\%) }\end{array}$ & $\begin{array}{l}\text { Total } \\
\mathbf{n} /(\%)\end{array}$ \\
\hline \multirow[t]{2}{*}{ Cabeza } & q & $21(5.7)$ & $6(1.6)$ & $\mathrm{NE}$ & $7(1.9)$ & $34(9.1)$ \\
\hline & $\hat{\sigma}$ & $5(1.3)$ & $5(1.3)$ & $\mathrm{NE}$ & $31(8.3)$ & $41(11.1)$ \\
\hline \multirow[t]{3}{*}{ Oreja } & q & $58(15.6)$ & $17(4.6)$ & $\mathrm{NE}$ & $8(2.2)$ & $83(22.3)$ \\
\hline & $\sigma^{1}$ & $11(2.9)$ & $11(2.9)$ & $\mathrm{NE}$ & 48 (12.9) & $70(18.8)$ \\
\hline & $\mathrm{N}$ & $\mathrm{NE}$ & $\mathrm{NE}$ & $21(5.7)$ & $\mathrm{NE}$ & $21(5.7)$ \\
\hline \multirow[t]{2}{*}{ Cuello } & q & $9(2.4)$ & $4(1.1)$ & $\mathrm{NE}$ & $5(1.3)$ & $18(4.8)$ \\
\hline & $\hat{0}$ & $1(0.3)$ & $5(1.3)$ & NE & $29(7.8)$ & $35(9.4)$ \\
\hline \multirow[t]{2}{*}{ Lomo } & q & $4(1.1)$ & $\mathrm{NE}$ & NE & $6(1.6)$ & $10(2.7)$ \\
\hline & $\hat{0}$ & $\mathrm{NE}$ & $\mathrm{NE}$ & $\mathrm{NE}$ & $31(8.3)$ & $31(8.3)$ \\
\hline \multirow[t]{2}{*}{ Patas } & q & $6(1.6)$ & $2(0.5)$ & $\mathrm{NE}$ & $2(0.5)$ & $10(2.7)$ \\
\hline & $0^{1}$ & NE & $3(0.8)$ & $\mathrm{NE}$ & $16(4.3)$ & $19(5.1)$ \\
\hline \multicolumn{2}{|c|}{$\begin{array}{l}\text { Total por } \\
\text { especie }(\mathrm{n} / \%)\end{array}$} & $115(30.9)$ & $53(14.2)$ & $21(5.7)$ & $183(49.2)$ & $372(100)$ \\
\hline
\end{tabular}

$\mathrm{N}=$ ninfas; $\mathrm{n}=$ número de ejemplares; $\mathrm{NE}=$ no se encontraron.

En el estado de Sonora se colectó el $54.8 \%$ (204/372) de las garrapatas de los dos hospederos; en $O$. virginianus el $9.3 \%$ (19/204) fueron ninfas de O. megnini y $0.98 \%(2 / 204)$ ninfas del mismo ectoparásito en $O$. canadensis junto con $89.7 \%$ de D. hunteri (Cuadro 1). En Nuevo León se colectaron $40.6 \%(151 / 372)$ garrapatas de $O$. virginianus con dos especies diferentes: $R$. microplus (98/151, $64.9 \%$ ) y D. nitens (34/151, 35.1\%). En Tamaulipas, en $O$. virginianus se colectaron únicamente 17 ejemplares de $R$. microplus, que representa el $4.56 \%$ (17/372) del total de garrapatas adultas en las tres localidades.

La distribución corporal de las garrapatas en los hospederos fue mayor en las orejas con un total de 174 (46.77\%) de las cuatro especies (22.3\% de hembras, $18.8 \%$ de machos y $5.7 \%$ ninfas) y en la parte superior de la cabeza (9.1\% hembras y $11.1 \%$ machos), seguida por el cuello, escapula y por último en las extremidades inferiores. Al analizar las diferentes especies de garrapatas y su distribución corporal, sin importar hospedero y localidad, presentaron asociación significativa $\left(\mathrm{X}^{2}=46.18\right.$, g.1.=8, $\left.P<0.05\right)$, aún en $O$. megnini las ninfas se localizaron exclusivamente en las orejas. También las hembras y machos de las 
diferentes especies de garrapatas se asociaron significativamente con la ubicación corporal $\left(\mathrm{X}^{2}=13.25\right.$, g.1.= 4, $\left.P<0.05\right)$.

En cuanto a la prevalencia, abundancia e intensidad de las garrapatas de $O$. virginianus, $R$. microplus presentó la mayor con el $30.9 \%, 0.49$ y 2.88, respectivamente; pero en $O$. canadensis, D. hunteri es la más sobresaliente (Cuadro 3).

Cuadro 3: Prevalencia, abundancia e intensidad de infestación por especie de garrapatas

\begin{tabular}{|c|c|c|c|c|c|c|}
\hline \multirow{2}{*}{ Especie } & \multicolumn{3}{|c|}{ O. virginianus $(40 / 233)$} & \multicolumn{3}{|c|}{ O. canadensis $(4 / 4)$} \\
\hline & Prev (\%) & Abund $\bar{X}$ & Intens & Prev \%) & Abund $\bar{X}$ & Intens \\
\hline R. microplus & $\begin{array}{l}115 \\
(30.9)\end{array}$ & 0.49 & 2.88 & -- & -- & -- \\
\hline D. nitens & $53(14.2)$ & 0.23 & 1.33 & -- & -- & -- \\
\hline O. megnini & $19(5.1)$ & 0.082 & 0.48 & $2(0.54)$ & 0.5 & 0.5 \\
\hline D. hunteri & -- & -- & -- & $183(49.2)$ & 45.8 & 45.8 \\
\hline
\end{tabular}

Prev $=$ prevalencia; Abund $=$ abundancia; Intens $=$ intensidad .

\section{Discusión}

Rhipicephalus (Boophilus) microplus conocida como garrapata del ganado, en $O$. virginianus de Nuevo León y Tamaulipas es la que presentó mayor prevalencia debido a que habitan el mismo territorio cinegético. Esta garrapata es considerada de mayor incidencia en los sistemas de producción ganadera, debido a las grandes pérdidas económicas que causa a nivel mundial $^{(27)}$ además de ser vector biológico de Babesia bovis, B. bigemina y Anaplasma marginale; en este estudio, se considera que $O$. virginianus juega un papel importante como reservorio natural de estas enfermedades en las zonas de estudio, tal como se ha reportado en áreas de Texas, EUA que limitan con Nuevo León y Tamaulipas ${ }^{(27)}$. Durante el control con insecticidas, las garrapatas hembras y larvas escapan hacia hábitats favorables para sobrevivir después del tratamiento, lo que facilita el recrudecimiento de las infestaciones en el ganado y de estos ungulados ${ }^{(28)}$. En cambio en los ejemplares de $O$. virginianus analizados en Sonora, no se encontró $R$. micropulus debido a que es un estado libre de esta garrapata, según el Servicio de Inspección de Sanidad Animal y Vegetal (APHIS) del Departamento de Agricultura de EUA (USDA), reportado por SADER/SENASICA ${ }^{(17)}$, pero en estados como Nuevo León y Tamaulipas, siguen los esfuerzos para su erradicación a pesar de que el programa opera una zona de cuarentena permanente en el sur de Texas, EUA, a lo largo de la frontera con México ${ }^{(28)}$. En el norte de México, el manejo del $O$. virginianus es a través de ranchos cinegéticos, debido a los ingresos que se perciben con los permisos de cacería, las pieles y la carne para consumo humano; en estos ranchos, $O$. virginianus en semi-cautiverio, comparten las mismas áreas de alimentación, bebida y traslados con el ganado bovino, por 
lo que esto puede ser un factor de riesgo de infestación y se torna difícil la erradicación de esta $\operatorname{garrapata}^{(29,30)}$.

La prevalencia de $R$. micropulus fue de $31 \%$ en el norte de México, menor que en Yucatán, México, donde se reportó un $97 \%$ en Cervus elaphus, que se considera como un importante hospedero competente de esta especie de garrapata, la cual, además de alimentarse, completa su ciclo de desarrollo ninfal ${ }^{(3)}$. Esta misma especie, también fue la más común en $O$. $v$. yucatanensis y Mazama temama, con una frecuencia del $28.4 \%$ y una intensidad de 25.2 de garrapatas por animal $^{(31)}$. En cambio, $R$. annulatus fue reportada exclusivamente en $C$. elaphus con una prevalencia de $7.9 \%$ en la provincia de Cádiz, España ${ }^{(11)}$.

En Nuevo León, se colectó $D$. nitens que hasta la fecha no ha sido reportada para el estado en $O$. virginianus, aunque existen reportes en varios estados del país, pero en diferentes hospederos como ganado, caballos, perros, mulas y roedores ${ }^{(32)}$. La importancia veterinaria de este ectoparásito es que las hembras de D. nitens transmiten Babesia caballi a su progenie trasováricamente, y todos sus estadios son competentes para esta enfermedad, además de ser agente causal de piroplamosis en caballos ${ }^{(33)}$.

En este estudio se reportaron ninfas de la garrapata espinosa (O. megnini) en $O$. virginianus $(5.1 \%)$ y $O$. canadensis $(0.53 \%)$ de Sonora, porque el estado adulto no es ectoparásito ${ }^{(34)}$, a pesar de que su distribución se extiende desde el suroeste de EUA hasta el sur de México y Suramérica, a esta ninfa no se les ha dado la misma importancia que a otras garrapatas Ixodidas, como $O$. megnini, ya que puede tener múltiples ingestas sanguíneas y depositar lotes de huevos que representan un peligro en el ámbito veterinario y clínico, por presentar una predilección por el canal auditivo, que puede resultar en una otoacariasis, con complicaciones de otitis externa, el $90 \%$ de los casos con dolor ótico, entre otros signos de otitis interna como son parálisis facial y/o respiratoria; afecta a las personas que mantienen un alto contacto operativo con animales de ganado, ya sean vacas, mulas, cabras, conejos y borregos $^{(35)}$. Este ectoparásito puede causar daño en el hospedero como severas irritaciones, pérdida de peso y puede afectar el comportamiento de las crías ${ }^{(36)}$. En ungulados y otros hospederos, cuando una garrapata o ninfa se alimenta causa pérdida de sangre lo que predispone a la atracción de otros insectos causándoles estrés en los hospederos ${ }^{(37)}$, además actúan como vectores de rickettsias causantes de fiebre manchada y Coxiella burnetii (fiebre $\mathrm{Q})^{(38)}$.

En Sonora, fue donde se encontró la mayor abundancia de garrapatas en borrego cimarrón, del total de garrapatas colectadas, el $49.19 \%$ pertenecieron a la especie D. hunteri y en machos tuvo mayor proporción con $41.6 \%$. Esta garrapata se ha observado casi exclusivamente en este hospedero, lo cual concuerda con otros autores y se tiene registrada también en Baja California en poblaciones silvestres de $O$. canadensis $^{(32,39)}$. En California USA, las poblaciones de este hospedero fueron seropositivas a Anaplasma spp $^{(40)}$, inclusive 
se considera como vector primario de A. ovis (Lestoquard, 1924) ${ }^{(41,42)}$, y otras rikettsias, lo que sugiere la importancia de este ectoparásito en la epidemiología de estas enfermedades ${ }^{(42)}$.

De acuerdo con la región corporal del hospedero, al tomar en cuenta el total de garrapatas, se observa que las cuatro especies de garrapatas tuvieron mayor predilección por las orejas, seguido de la cabeza. En Capreolus capreolus, el $61 \%$ de las garrapatas prefirieron zonas en la cabeza ${ }^{(12,21)}$, con un $32.02 \%$ de garrapatas en ganado en la combinación con orejas y cabeza y a su vez, en ovejas relacionaron la predilección alimenticia en cabeza que incluye las orejas con el $48.08 \%$, lo cual es consistente con los resultados encontrados en el presente estudio. La preferencia de estos ectoparásitos por la cabeza y las orejas sobre la posición del hospedero se relaciona por grosor de la piel que es relativamente delgada y vascularizada en ambas regiones corporales ${ }^{(43,44)}$.

En O. canadensis, los machos tuvieron una proporción similar para cada región corporal, con acentuación en las orejas y cabeza. Los resultados fueron similares en un estudio de regiones tropicales de México, la distribución de las garrapatas de la familia Ixodidae en la infestación de las ovejas fue de $26.50 \%$ en cabeza y cuello ${ }^{(45)}$. Se ha reportado que la densidad de garrapatas en $O$. virginianus puede ser muy variable, y que esta depende de la época estacional en que se colecte y la edad de los ungulados ${ }^{(12,46)}$. En este estudio se colectó en la temporada otoño-invierno justo cuando se permite la cacería coincidente con que las garrapatas fueran adultas, a excepción de O. megnini, en la cual su estado parasítico fue ninfal. En las garrapatas del género Ixodes y Dermacentor presentaron una preferencia hacia los ungulados más jóvenes, debido a sus hábitos y al grosor de la piel más delgada en Capreolus capreolus, además las garrapatas adultas no tuvieron preferencia sobre el sexo del hospedero pero sí por su masa corporal ${ }^{(12,46)}$. Aunque en el sur de Texas, EUA, donde existe un área de cuarentena de erradicación de garrapatas en el ganado, no se logran erradicar por movimientos no regulados de ganado ilegal y la dispersión de animales de vida silvestre como $O$. virginianus en México(27).

\section{Conclusiones e implicaciones}

Este trabajo reporta la presencia de cuatro especies de garrapatas en $O$. virginianus ( $O$. megnini, $R$. microplus y D. nitens) y dos en O. canadensis (O. megnini y D. hunteri) de ranchos cinegéticos del norte de México, las cuales juegan un papel crítico en la epizootiología de patógenos ${ }^{(4)}$, considerándose necesario realizar más estudios que identifiquen a potenciales vectores de enfermedades y microorganismos patógenos asociados a las garrapatas de los ungulados de importancia cinegética en el país y determinar el control de los mismos. La región corporal más infestada fueron las orejas y las menos infestadas fueron las piernas como respuesta al grosor de la piel en estas regiones corporales. Una de las principales estrategias para la erradicación de las garrapatas, es el conocimiento por la 
especificidad del hospedero como $O$. virginianus y otros ungulados, en el cual se recomendaría dispersar acaricidas, ivermectinas específicas según el tipo de suelo o pastizal $^{(48)}$, además de tomar en cuenta su ciclo de vida, ya que podría variar la susceptibilidad al insecticida ${ }^{(37)}$. Por consiguiente, el conocimiento de la distribución de garrapatas en el norte de México, su abundancia e intensidad de las mismas sobre $O$. virginianus y $O$. canadensis, ayudará a implementar medidas preventivas o control en ranchos cinegéticos y en la ganadería, así como en la importación o caza de estos ungulados, además de prevenir el desarrollo de nuevos vectores que pueden ser causantes potenciales de enfermedades infecciosas de salud pública y zoonótica.

\section{Agradecimientos}

Al grupo OVIS, S.A. DE C.V. por su participación en la colecta de garrapatas. A CONACyT por la beca de Maestría en Ciencias otorgada al primer autor.

\section{Literatura citada:}

1. Klompen JSH, Black WC, Keirans JE, Oliver Jr JH. Evolution of Ticks. Annu Rev Entomol 1996;41:141-161. doi:10.1146/annurev.en.41.010196.001041.

2. García-Vázquez Z. Garrapatas que afectan al ganado bovino y enfermedades que trasnmiten en México. 1er. Simposium de Salud y Producción de Bovinos de Carne en la Zona Norte-Centro de México 2010;1-9. http://biblioteca.inifap.gob.mx:8080/jspui/handle/123456789/3281 Consultado 5 Jun, 2019.

3. Rodríguez-Vivas RI, Ojeda-Chi MM, Rosado-Aguilar JA, Trinidad-Martínez IC, TorresAcosta JFJ, Ticante-Perez V, et al. Red deer (Cervus elaphus) as a host for the cattle tick Rhipicephalus microplus (Acari: Ixodidae) in Yucatan, Mexico. Exp Appl Acarol 2013;60:543-552. doi:10.1007/s10493-013-9672-z.

4. Koneman E, Koneman AS: Diagnostico microbiologico/Texto y atlas en color. 6ta ed. Buenos Aires, Argentina: Editorial Médica Panamericana; 2008.

5. Claerebout E, Losson B, Cochez C, Casaert S, Dalemans AC, De Cat A, et al. Ticks and associated pathogens collected from dogs and cats in Belgium. Parasit Vectors 2013;6:183. doi:10.1186/1756-3305-6-183.

6. Sosa-Gutiérrez G, Vargas M, Torres J. Gordillo-Pérez G. Tick-borne rickettsial pathogens in rodents from Mexico. J Biomed Sci Eng 2014;7:884-889. doi:10.4236/jbise.2014.711087. 
7. CDC. Centers for Disease Control and Prevention. New \& Emerging Tickborne diseases: $\begin{array}{lllll}\text { Agents, clinical } & \text { features } & 2018 .\end{array}$ https://www.cdc.gov/ticks/diseases/trends.html\#2013-video Accessed 12 Sept, 2018.

8. Bush JD, Stone NE, Nottingham R, Araya-Anchetta A, Lewis J, Hochhalter C, et al. Widespread movement of invasive cattle fever ticks (Rhipicephalus microplus) in southern Texas leads to shared local infestations on cattle and deer. Parasit Vectors 2014;7:188. doi:10.1186/1756-3305-7-188.

9. Shaw MT, Keesing F, McGrail R, Ostfeld RS. Factors influencing the distribution of larval blacklegged ticks on rodent hosts. Am J Trop Med Hyg 2003;68:447-452.

10. Blagburn BL, Dryden MW. Biology, treatment, and control of flea and tick infestations. Vet ClinNorth Am Small Anim Pract 2009;39:1173-1200. doi:10.1016/j.cvsm.2009.07.001.

11. Ruiz-Fons F, Fernandez de Mera IG, Pelayo-Acevedo UH, Höfle U, Vicente J, De la Fuente $\mathrm{J}$, et al. Ixodid ticks parasitizing Iberian red deer (Cervus elaphus hispanicus) and European wild boar (Sus scrofa) from Spain: Geographical and temporal distribution Vet Parasitol 2006;140:133-142. doi:10.1016/j.vetpar.2006.03.033.

12. Vor T, Kiffner C, Hagedorn P, Niedrig M, Ru F. Tick burden on European roe deer (Capreolus capreolus). Exp Appl Acarol 2010;51:405-417. doi:10.1007/s10493-0109337-0.

13. Martínez Arzate SG. Análisis filogenético molecular de la secuencia de la proteína Bm86 de la garrapata Rhipicephalus (boophilus) microplus de Colima, México [tesis maestria]. Toluca, Estado de México. Universidad Autónoma del Estado de México; 2014.

14. SENASICA. Situación actual del control de la garrapata Boophilus spp. 2016. https://www.gob.mx/senasica/documentos/situacion-actual-del-control-de-lagarrapata-boophilus-spp. Consultado 20 Sept, 2019.

15. Rodríguez Vivas RI, Grisi L, Pérez de León A, Silva Villela H, Torres Acosta J, Fragoso $\mathrm{H}$, et al. Potential economic impact assessment for cattle parasites in Mexico. Review. Rev Mex Cienc Pecu 2017;8(1):61-74.

16. OVIS. PROGRAMAS.2011. http://ovis.org.mx/programas/ Consultado 10 Nov, 2017.

17. SEMARNAT. Reglamento de la ley general de vida silvestre. Diario Oficial de La Federación; 2014; DOF 09-05-2014. 1-52. http://biblioteca.semarnat.gob.mx/janium/Documentos/Ciga/agenda/DOFsr/DO2008.p df. Consultado 15 Abr, 2018. 
18. SEMARNAT. Registros de unidades de manejo para la conservación de la vida silvestre (UMA). 2019. https://datos.gob.mx/busca/dataset/registros-de-unidades-de-manejopara-la-conservacion-de-la-vida-silvestre-uma/resource/8815e80b-1779-469f-80b8b4a4756f61c3.

19. INEGI. Cuentame. 2017. http://www.inegi.org.mx/ http://www.cuentame.inegi.org.mx/monografias/informacion/son/territorio/div_munici pal.aspx?tema=me\&e=26. Consultado 21 Mar, 2018.

20. OCDE. (2018). Nuevo Laredo, Tamaulipas | OCDE Mexico. http://www.ocdemexico.org.mx/Tamaulipas/Nuevo-Laredo/. Consultado Mar 25, 2018.

21. Warwick BT, Bak E, Baldassare J, Gregg E, Kioko J, Saning K, et al. Abundance estimations of ixodid ticks on Boran cattle and Somali sheep in Northern Tanzania. Int J Acarol 2016;42:12-17. doi:10.1080/01647954.2015.1109708.

22. Amerasinghe F, Breisch N, Azad A. Distribution, density, and Lyme disease spirochete infection in Ixodes dammini (Acari: Ixodidae) on white-tailed deer in Maryland. J Med Entomol 1992;29:54-61.

23. Keirans JE, Litwak TR. Pictorial key to the adults of hard ticks, family Ixodidae (Ixodida: Ixodoidea), east of the Mississippi River. J Med Entomol 1989;26:435-448.

24. Delabra G, Fragoso H, Franco R, Martínez F, Ortiz M, Ortiz A, et al. Manual de identificación de las especies de garrapatas de importancia en México. México: Dirección General de Salud Animal. Secretaría de Agricultura, Ganadería y Desarrollo Rural. 1996.

25. Walker AR, Bouattor A, Camicas J, Estrada-Pena, Horak IG, Latiff A, et al. Ticks of domestic animals in Africa, a guide to identification of species. Edinburgh Scotland, U.K: Bioscience Reports. 2014.

26. Bush AO, Lafferty KD, Lotz JM, Shostak AW. Parasitology meets ecology on its own terms: Margolis et al. revisited. J Parasitol 1997;83(4):575-583.

27. Busch JD, Stone NE, Nottingham R, Araya-Anchetta A, Lewis J, Hochhalter, et al. Widespread movement of invasive cattle fever ticks (Rhipicephalus microplus) in southern Texas leads to shared local infestations on cattle and deer. Parasit Vectors $2014 ; 7: 188$.

28. Wang HH, Teel PD, Grant WE, Schuster G, Pérez de León AA. Simulated interactions of white-tailed deer (Odocoileus virginianus), climate variation and habitat heterogeneity on southern cattle tick (Rhipicephalus (Boophilus) microplus) eradication methods in south Texas, USA. Ecol Modell 2016;342:82-96. 
29. Cantú-Martinez M, Salinas-Meléndez JA, Zarate-Ramos J, Ávalos-Ramírez R, MartínezMuñoz A, Segura-Correa J. Prevalence of antibodies against Babesia bigemina and $B$. bovis in white-tailed deer (Odocoileus virginianus texanus) in farms of northeastern Mexico. J Anim Vet Adv 2008;7:121-123.

30. Medrano C, Boadella M, Barrios H, Cantú A, García Z, De la Fuente J, et al. Zoonotic pathogens among white-tailed deer, northern Mexico, 2004-2009. Emerg Infect Dis 2012;18:1372-1374.

31. Ojeda-Chi M, Rodriguez-Vivas RI, Esteve-Gasent MD, Pérez de León A, Modarellid J J, Villegas-Perez S. Molecular detection of rickettsial tick-borne agents in white-tailed deer (Odocoileus virginianus yucatanensis), mazama deer (Mazama temama), and the ticks they host in Yucatan, Mexico. Ticks Tick Borne Dis 2019;10:365-370. doi: 10.1016/j.ttbdis.2018.11.018

32. Guzmán-Cornejo C, Robbins RG, Guglielmone AA, Montiel-Parra G, Rivas G, Pérez TM. The Dermacentor (Acari, Ixodida, ixodidae) of Mexico: Hosts, geographical distribution and new records. ZooKeys 2016;569:1-22. doi:10.3897/zookeys.569.7221.

33. Schwint ON, Knowles DP, Ueti MW, Kappmeyer LS, Scoles GA. Transmission of Babesia caballi by Dermacentor nitens (Acari: Ixodidae) is restricted to one generation in the absence of alimentary reinfection on a susceptible equine host. J Med Entomol 2008;45:1152-1155. doi:10.1603/0022-2585(2008)45[1152:TOBCBD]2.0.CO;2.

34. Nava S, Mangold J, Guglielmone AA. Field and laboratory studies in a Neotropical population of the spinose ear tick, Otobius megnini. Med Vet Entomol 2009;23:1-5. doi:10.1111/j.1365-2915.2008.00761.X

35. Cakabay T, Gokdogan O, Kocyigit M. Human otoacariasis : Demographic and clinical outcomes in patients with ear-canal ticks and a review of literature. J Otol 2016;11:111117. doi:10.1016/j.joto.2016.06.003.

36. Niebuhr CN, Mays SE, Breeden JB, Lambert BD, Kattes DH. Efficacy of chemical repellents against Otobius megnini (Acari :Argasidae) and three species of ixodid ticks. Exp Appl Acarol 2014;64:99-107. doi:10.1007/s10493-014-9799-6.

37. Almada Resende JDS, Daemon E, De Olivera Montero CM, Maturano R, Prata DA, Rodrigues AFS. Toxicity of solvents and surfactants to Amblyomma cajennense (Fabricius, 1787) (Acari:Ixodidae) and Dermacentor nitens (Neumann, 1897) (Acari : Ixodidae) larvae. Exp Parasitol 2012;131:139-142. doi:10.1016/j.exppara.2012.03.002.

38. Diyes GCP, Rajakaruna RS. Seasonal dynamics of spinose ear tick Otobius megnini associated with horse otoacariasis in Sri Lanka. Acta Trop 2016;159:170-175. doi:10.1016/j.actatropica.2016.03.025. 
39. Crosbie P, Goff W, Stiller D, Jessup D. The distribution of Dermacentor hunteri and Anaplasma sp. in desert bighorn sheep (Ovis canadensis). Med Vet Entomol 1997;83:31-37.

40. De la Fuente J, Atkinson MW, Hogg JT, Miller DS, Naranjo V, Almazán C, et al. Genetic characterization of Anaplasma ovis strains from bighorn sheep in Montana. J Wildl Dis 2006;42:381-385. doi:10.7589/0090-3558-42.2.381.

41. Stiller D, Crosbie PR, Boyce WM, Goff WL. Dermacentor hunteri (Acari: Ixodidae): an experimental vector of Anaplasma marginale and A. ovis (Rickettsiales:Anaplasmataceae) to calves and sheep. J Med Entomol 1999;36:321-324.

42. Yabsley MJ, Davidson WR, Stallknecht DE, Varela AS, Swift PK, Devos JC, et al. Evidence of tick-borne organisms in mule deer (Odocoileus hemionus) from the Western United States. Vector Borne Zoonotic Dis 2005;5:351-362.

43. Bloemer SR, Zimmerman RH, Fairbanks K. Abundance, attachment sites, and density estimators of lone star ticks (Acari:Ixodidae) infesting white-tailed deer. J Med Entomol 1988;25:95-300. doi:10.1093/jmedent/25.4.295.

44. L'Hostis M, Diarra O, Seegers H. Sites of attachment and density assessment of female Ixodes ricinus (Acari:Ixodidae) on dairy cows. Exp Appl Acarol 1994;18:681-689. doi:10.1007/BF00051535

45. Coronel-Benedett KC, Ojeda-Robertos NF, Gonzalez-Garduño R, Martinez- Ibañez F, Rodriguez-Vivas RI. Prevalence, intensity and population dynamics of hard ticks (Acari:Ixodidae) on sheep in the humid tropics of Mexico. Exp Appl Acarol 2018:74:99-105.

46. Kiffner C, Lödige C, Alings M, Vor T, Rühe F. Body-mass or sex-biased tick parasitism in roe deer (Capreolus capreolus). A GAMLSS approach. Med Vet Entomol 2011;25:39-44. doi:10.1111/j.1365-2915.2010.00929.x

47. Han S, Hickling GJ, Tsao JI. High Prevalence of Borrelia miyamotoi among adult blacklegged ticks from white-tailed deer. Emerg Infect Dis 2016;22: 22-24.

48. Pound JM, George JE, Kammlah DM, Lohmeyer KH, Davey RB. Evidence for role of white-tailed deer (Artiodactyla: Cervidae) in epizootiology of cattle ticks and Southern cattle ticks (Acari:Ixodidae) in reinfestations along the Texas/Mexico border in South Texas: A Review and Update. J Econ Entomol 2010;103:211-218. doi:10.1603/EC09359 